\title{
The Inhibitory Effect of Ileal Mucosal Media Originated from FVB/N mice strain on Escherichia coli LF82 Invasion
}

\author{
(D) Hüsamettin AYGÜN11, id Murat KARAMEŞE2 , iD Fikret UYAR1
}

${ }^{1}$ Dicle University Faculty of Science, Department of Biology, Diyarbakır, Turkey

${ }^{2}$ Kafkas University Faculty of Medicine, Deparment of Medical Microbiology, Kars, Turkey

\begin{abstract}
Objective: The aim of this study was to investigate the effect of healthy mucosa on adhesive and invasive properties of AIEC reference strain Escherichia coli (E. coli) LF82. For this purpose, we had designed special medias that contained cell culture medium and mucosal content obtained from different regions (colon and ileum) of the digestive tract.

Methods: We tested the infecting ability of AIEC reference strain E. coli LF82 on I-407 cells in the presence of mucosal media (Muc-M) under in vitro conditions. Muc-M composed of certain rates of cell culture medium or M63 minimal medium and mucosal contents obtained from different part of intestine were designed for cell-infection experiments and biofilm-formation assays.

Results: The result showed that the mucosal media decreased the infection percentage of E. coli LF82 strain when compared with control group. It was seen that the mucosal media originating from ileum almost completely inhibited the invasion of LF82 strain. On the other hand, it was observed that the mucosal media prepared from colon) reduced the bacterial invasion only in half the rate when compared with control.

Conclusion: The findings showed that these medias obtained from different regions of the intestinal tract affected LF82 invasion at different rates. Therefore, this study provided crucial information that could contribute to the future studies on the localization of bacteria
\end{abstract}

Keywords: Escherichia coli LF82, Crohn's disease, biofilm, pathobiont, adhesion, invasion

\section{Introduction}

Crohn's disease $(\mathrm{CD})$ is a chronic inflammatory bowel disease that can affect various parts of the intestinal tract and complex genetic and environmental factors are involved in its development. On the other hand, the intestinal flora plays an important role in the development of this disease because there are some strong findings about the role of the intestinal flora in the development of Crohn's disease. One of these is the detection of the significant inflammatory process in response to the contact of luminal content with terminal ileum, shown after surgical operations in Crohn's patients. However, when the fecal flow is directed to another side, the signs of healing are observed in these regions (1). And the others are the positive results of antibiotic treatment in some Crohn's patients, the reduction of ulceration symptoms following antibiotic treatment in multiple animal experiments and no findings about colitis in germ-free animals (2).

On the other hand, scientific studies performed about the relationship between intestinal flora and CD have shown the presence of a significant increase in the amount of Escherichia coli (E. coli) in individuals with the ileal type of CD when compared to normal individuals (3-5). After some detailed studies, a new E. coli member, adherent-invasive (AIEC), was isolated from the inflamed tissue of an individual with ileal type of CD (6). In vitro studies have shown that this new strain can adhere to and invade intestinal epithelial cells. Similarly, it has been determined by in vitro methods that this strain can live and multiply in

Address for Correspondence: Murat KARAMEŞE, Kafkas University Faculty of Medicine, Deparment of Medical Microbiology, Kars, Turkey

Received: 04.06.2018

Phone: +90 5548638853 E-mail: murat_karamese@hotmail.com ORCID ID: orcid.org/0000-0001-7803-

Accepted: 17.10 .2018 1462

Cite this article as: Aygün H, Karameşe M, Uyar F. The Inhibitory Effect of Ileal Mucosal Media Originated from FVB/N mice strain on Escherichia coli LF82 Invasion. Bezmialem Science 2019;7(3):198-203. 
macrophages and cause a high amount of tumour necrosis factor-alpha (secretion. These bacteria was also shown to cause high-level expression of carcino-embryonic antigen-related cell adhesion molecule- 6 molecules, a kind of adhesion receptor in patients with CD (7).

Despite all the research done with AIEC, it is still unclear how these bacteria play a role in the development of Crohn's disease. Furthermore, it is not possible to determine the location of these bacteria in the intestinal flora because these bacterial specific molecules have not yet been identified. The intestinal mucus covering the intestinal epithelium along the digestive tract serves as a barrier to pathogenic microorganisms reaching the epithelial cells as well as hosting many commensal bacteria (8).

The basic structure of the mucus is composed of mucins which are a family of high molecular weight, heavily glycosylated proteins (glycoconjugates) produced by epithelial tissues in most animals. Typically, these mucins are glycoproteins containing $80 \%$ carbohydrate and consisting of proline, serine and threonine amino acid repeats (referred to as peroxisomal targeting signal sequence) $(8,9)$. Although there are several types of mucin glycoproteins that form the mucous membrane throughout the digestive tract, goblet cells secrete MUC-2 mucin in the colon and small intestine of the intestinal tract. However, the same mucin exhibits different behaviors in the small intestine and the colon (10-12). Studies suggest that this difference originates from the differences in glycan epitopes in MUC-2. It is emphasized that these oligosaccharide units present in the mucus may also play an important role in the distribution of the intestinal flora in the digestive tract (13). Therefore, the mucin glycoprotein in different compartments of the digestive tract may have a key role in studies on the localization of AIEC bacteria, or on the detection of its specific molecules.

The aim of current study was to investigate the effect of healthy mucosa on adhesive and invasive properties of AIEC. For this purpose, we designed special medias that contained cell culture medium and mucosal content obtained from different regions (colon and ileum) of the digestive tract.

\section{Methods}

\section{Bacterial Strain}

The AIEC reference strain, E. coli LF82 isolated from an ileal lesion of a patient with CD was kindly provided by Dr. Nicolas Barnich and Dr. Elisabeth Billard (Universite' d'Auvergne, Clermont-Ferrand, France). Bacteria were grown routinely in the Luria-Bertani (LB) broth overnight at $37{ }^{\circ} \mathrm{C}$ and without shaking.

\section{Cell Line and Cell Culture Procedure}

The intestine-407 (I-407) cell line (The Global Bioresource Center, chemokine (C-C motif) ligand 6, Manassas, VA, USA) was also kindly provided by Dr. Nicolas Barnich and Dr. Elisabeth Billard (Universite' d'Auvergne). The cells were maintained in an atmosphere containing $5 \% \mathrm{CO}_{2}$ at $37^{\circ} \mathrm{C}$ in Eagle minimum essential medium (MEM) (MEM; Sigma-Aldrich, MO, USA) supplemented with $10 \%(\mathrm{v} / \mathrm{v})$ heat- inactivated fetal bovine serum [Fetal bovine serum (FBS); Life Technologies, CA, USA], $1 \%$ nonessential amino acids (Life Technologies), 1\% L-glutamine (Life Technologies), 100,000 units/L penicillin, $100 \mathrm{mg} / \mathrm{L}$ streptomycin, $25 \mathrm{mg} / \mathrm{L}$ amphotericin $\mathrm{B}$ and $1 \%$ MEM vitamins solution X-100 (Life Technologies).

\section{Mice Strain}

FVB/N female mice were housed under specific pathogen-free conditions in the animal care facility of Universite' d'Auvergne (Clermont-Ferrand, France). Animal protocols were performed according to the local ethical rules during ERASMUS+ Traineeship Program between $23^{\text {th }}$ and June $19^{\text {th }} 2015$ in Universite' d'Auvergne (Clermont-Ferrand, France).

\section{Isolation of Ileum \& Colon and Preparation of Mucosal Media (Muc-M)}

Mucosal media (Muc-M) was designed as an artificial medium, which was isolated from the 8- to 10 -week-old female healthy $\mathrm{FVB} / \mathrm{N}$ mice intestine. Muc-MIR is originated from ileum regions and Muc-MCR is originated from colon regions of healthy mice intestines. The 8- to 10 -week-old female FVB/N mice were euthanized by cervical dislocation. The mouse was opened by ventral midline incision under $70 \%$ ethanol anesthesia. Colon was isolated as the distance $(\sim 5 \mathrm{~cm})$ from rectum to cecum. Then, the ileum was isolated from cecum to $(\sim 8 \mathrm{~cm})$ superior region of cecum. The colon and ileum regions of intestine were separately collected in sterile Petri dishes containing physiological water. The colon was brought into the open using scissors and the inner surface of the colon scrapped by a glass slide. This procedure was repeated also for ileum. Scrapped contents obtained from three mice were collected in a sterile $2 \mathrm{~mL}$ eppendorf tube for each region.

Cell infection procedure and biofilm assays were performed in different times. For cell-infection experiments, the tubes were labelled then, $1 \mathrm{~mL}$ sterile MEM added into tubes. Samples were treated for $15 \mathrm{~min}$ at $+4^{\circ} \mathrm{C}$ at a maximum speed of the disruptor. After disruption, samples were centrifuged for $10 \mathrm{~min}$ at $+4^{\circ} \mathrm{C}$ in 10,600x g. Supernatant was collected and transferred into falcon tubes separately for colonic regions and ileal regions.

Sterilization was performed by filtration method in the laminar air-flow cabinet. Samples were passed through filter with $0.45 \mu \mathrm{m}$ to new sterile falcons, then the filtrate was passed through filter with $0.20-\mu \mathrm{m}$ pore diameter to another new sterile falcons and finally, it was diluted with sterile MEM as half of their total volume. The mentioned procedures were similarly performed for biofilm-formation assays. The only difference between two methods is that M63 minimal medium supplemented with $8 \mathrm{~g} / \mathrm{L}$ $(0.8 \%)$ glucose was used instead of MEM for biofilm-formation assay. To test the sterility of these media, Muc- $M$ were inoculated onto LB and Mac Conkey (Oxoid, Basingstoke, UK) agar plates, and afterward, it was seen no colony forming on both plates after overnight incubation at $37^{\circ} \mathrm{C}$. 
By the way, the effects of Muc-M alone on I-407 cell monolayers were also tested for cell infection experiments with a similar experiment performed by Aygun et al (14). Briefly, I-407 cell monolayers in the cell culture medium including $20 \%$ of Muc-M was incubated for $3-4 \mathrm{~h}$ at $37^{\circ} \mathrm{C}$ with $5 \% \mathrm{CO}_{2}$. After incubation period, it was seen that the cell culture medium including 20\% of Muc-M had no negative or deleterious effects on cell monolayers. Finally, Muc-M was ready for cell infection and biofilm-formation assays.

\section{Cell Infectivity and Adhesion\&Invasion Experiments}

The cell infectivity, adhesion and invasion experiments were performed according to Darfeuille-Michaud method (15). I-407 cells were seeded in 24-well tissue culture plates (Sigma-Aldrich) at a density of $4 \times 10^{5}$ cells per well and incubated for $20 \mathrm{~h}$. The cell monolayers were washed twice with phosphate-buffered saline (PBS) ( $\mathrm{pH}$ 7.2). $200 \mu \mathrm{L}$ of Muc-M was prepared with MEM, was added to monolayers and brought to $1 \mathrm{~mL}$ with $10 \%$ FBSsupplemented MEM for each regions. 10\% FBS-supplemented MEM was only designed and used as control. Each monolayer was infected with a multiplicity of infection (MOI) of 10 bacteria per epithelial cell (MOI: 10). In $3 \mathrm{~h}$ incubation period, it was performed at $37^{\circ} \mathrm{C}$ with $5 \% \mathrm{CO}_{2}$.

For adhesion assay, after $3 \mathrm{~h}$ incubation period at $37^{\circ} \mathrm{C}$ with $5 \%$ $\mathrm{CO}_{2}$, the monolayers were washed three-times with PBS and the epithelial cells were then lysed with 1\% Triton X-100 (SigmaAldrich) in deionized water. The samples were diluted and plated onto LB agar plates to determine the number of colony forming unit (CFU), and the mean number of bacteria per cell was determined. Adhesion assays were performed in triplicate.

For invasion assay, after $3 \mathrm{~h}$ incubation period at $37^{\circ} \mathrm{C}$ with $5 \%$ $\mathrm{CO}_{2}$, the monolayers were washed three-times with PBS. One hundred $\mu \mathrm{L} / \mathrm{m}$ fresh culture medium containing gentamicin (Sigma-Aldrich) was added to cell media to kill the extracellular bacteria and $1 \mathrm{~h}$ incubation was performed. After incubation for an additional hour, monolayers were washed with PBS and $1 \%$ Triton X-100 in deionized water placed in the wells to lysis the eukaryotic cells for $5 \mathrm{~min}$. The samples were diluted and plated onto LB agar plates to determine the number of CFU. Invasive ability of LF82 with I-407 cell lines were expressed as the percentage of intracellular bacteria compared with the initial inoculum, taken as $100 \%$. Invasion assays were performed in triplicate.

\section{Statistical Analysis}

One-way ANOVA followed by Dunnet T3 test was used for the assessment of the numerical data. $P$ values $\leq 0.05$ were considered statistically significant. All assays were performed at least threetimes in separate experiments. Microsoft Excel and SPSS version 24.0 (IBM, NY, USA) programs were used for statistical analysis.

\section{Results}

We evaluated the effects of sterile mucosal media, derived from different parts of healthy intestines of FBV/N mice strain, on LF82 adhesion and invasion to human intestinal epithelial cell line (I-407) by adding each Muc-M (20; Muc-MCR or Muc-
MIR) to the cell culture medium before infection and during $3 \mathrm{~h}$ infection period for adhesion/invasion assays.

After incubation period, it was seen that the cell culture medium including $20 \%$ of Muc-M had no negative or deleterious effects on cell monolayers (Figure 1, Figure 1a, Figure 1b, Figure 1c and Figure 1d).

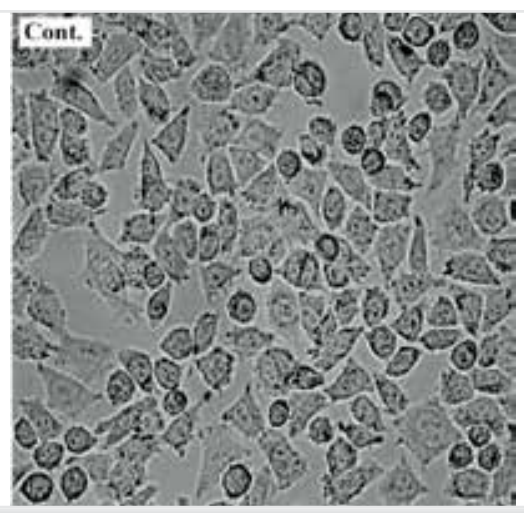

Figure 1. The apperance of cells in normal cell culture medium.

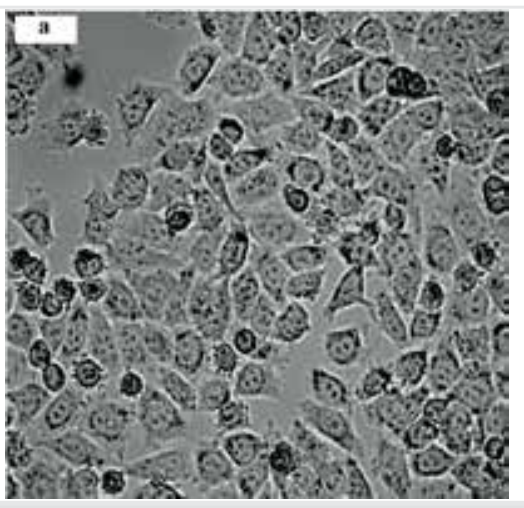

Figure 1a: Micrographs of crystal violet stained biofilms in the different mucosal medias originated from colon or ileum. The appearance of cells in mucosal media (Muc-MCR) after 3 hour incubation

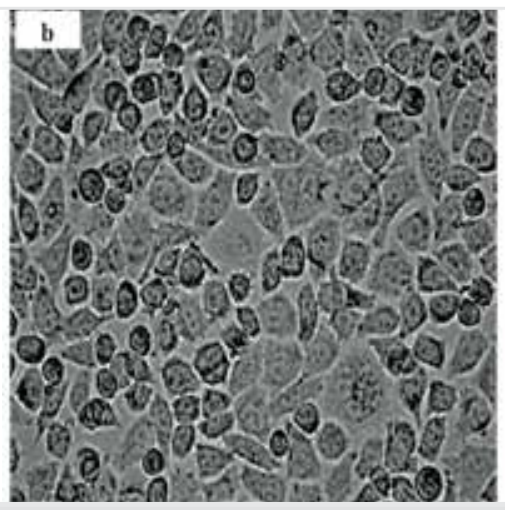

Figure 1b. The apperance of cells following 3- hour incubation, after replacement mucosal media (Muc-MCR) with fresh cell culture medium 


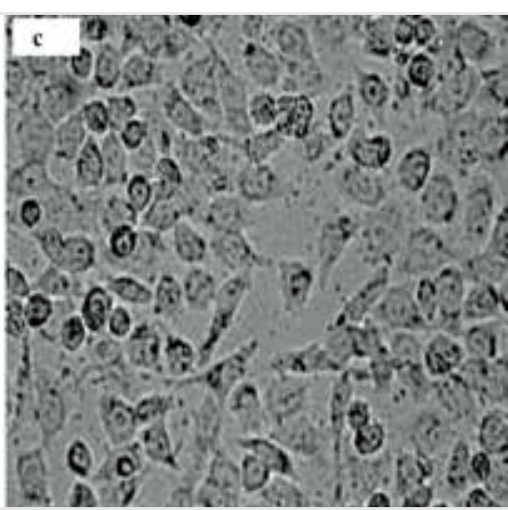

Figure 1c: Micrographs of crystal violet stained biofilms in the different mucosal medias originated from colon or ileum. Cell culture in mucosal media (Muc-MIR) after 3 hour incubation

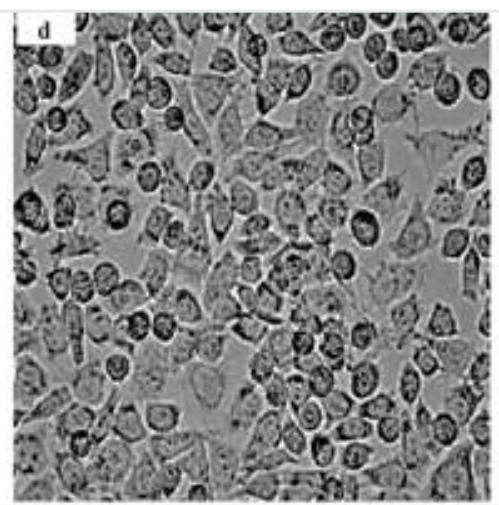

Figure 1d. The apperance of cells following 3- hour incubation, after replacement mucosal media (Muc-MIR) with fresh cell culture medium

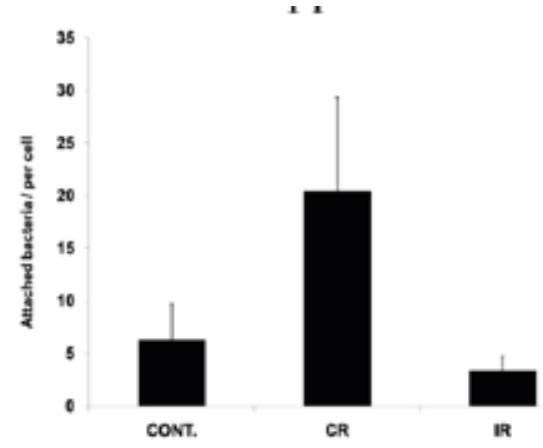

Figure 2. Adhesion abilities of adherent-invasive Escherichia coli LF82 in different medias including mucosal media originated from colon or ileum regions. Cell-associated bacteria were quantified after a 3-h infection period. Results are expressed as cell-associated (adherent+intracellular) bacteria per cell. ANOVA was used for multiple comparisons. CONT.: Control, CR: Colon region, IR: Ileum region

Adhesion results showed that the Muc-MIR medium almost completely inhibited LF82 invasion ( $\mathrm{p}<0.001)$, while the MucMCR medium reduced the LF82 invasion by $50 \%$ compared to the control, however this decline was not statistically significant ( $p>0.001)$ as seen in Figure 2. LF82 exhibited a higher adherent

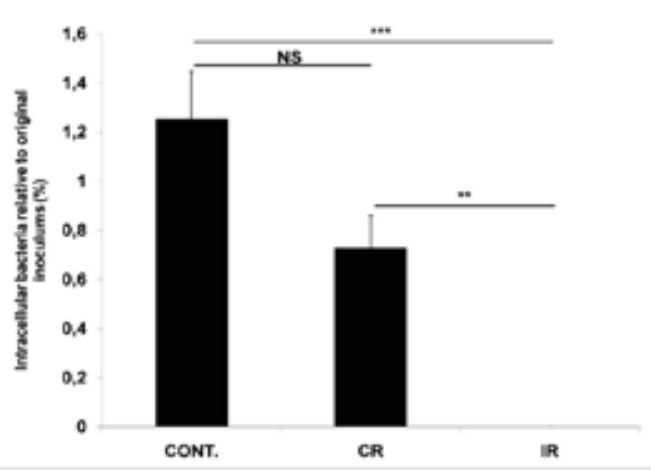

Figure 3. Invasion abilities of adherent-invasive Escherichia coli LF82 in different medias including mucosal media originated from colon or ileum regions. Cell-associated bacteria were quantified after a 3-h infection period. Results are expressed as cell-associated (adherent+intracellular) bacteria per cell. ANOVA was used for multiple comparisons. CONT.: Control, CR: Colon region, IR: Ileum region

ability to I-407 cell monolayers in cell culture medium including Muc-MCR than those including Muc-MIR $(\mathrm{p}<0.01$; as attached

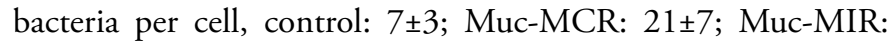
$4 \pm 1$; Figure 2). On the other hand, after gentamicin treatment following extra $1 \mathrm{~h}$ incubation period, LF82 invasion significantly decreased $(\mathrm{p}<0.05)$ in the cell culture media including $20 \%$ of Muc-MCR or Muc-MIR.

The results of invasion assay showed that both of these medias considerably reduced bacterial invasion (Figure 3). It was quite remarkable that the Muc-MIR medium originating from the ileum inhibited more LF82 invasion than the Muc-MCR medium prepared from the colon region of intestine $(\mathrm{p}<0.01$; intracellular bacteria relative to original inoculums (\%), control:

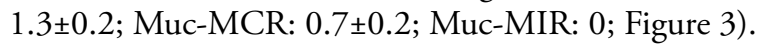

\section{Discussion}

Many studies examined the relationship between etiopathogenesis of CD and AIEC strains which are found in high amounts in the inflamed ileal tissues of patients with Crohn's disease. However, the current literature does not have enough evidence about the role of AIEC on Crohn's disease. The same studies showed that although the $E$. coli strains are relatively found in higher amounts in ileal tissues both in healthy and CD patients, the localization of AIEC in digestive tract has not yet been identified $(16,17)$. Therefore, studies to find these bacterial specific molecules will help illuminate the role of AIEC strains in the progression of CD. In the current study, we designed special medias that contained mucosal content obtained from different regions (colon and ileum) of FBV/N healthy female mice digestive tract. Then, we tested the infecting ability of AIEC reference strain $E$. coli LF82 to I-407 cells in the presence of Muc-M originating from the colon (Muc-MCR) and ileum (Muc-MIR) regions, under in vitro conditions.

After adding Muc-MCR or Muc-MIR media to the cell culture medium at $20 \%$ percentage, we infected the cells with LF82 bacteria. There was no statistically significant difference when we 
examined the results of the adhesions after the 3 hour infective period. However, the results of invasion assay showed that both of these media considerably reduced bacterial invasion. According to our results; the Muc-MIR medium almost completely inhibited LF82 invasion $(\mathrm{p}<0.001)$, while the Muc-MCR medium reduced the LF82 invasion by $50 \%$ compared to the control, however this decline was not statistically significant $(p>0.001)$. The results we obtained from the current study strongly supported our previous study that performed with Balb/c mouse strain (14). The strong inhibition of bacterial invasion, in particular, may be due to glycosylated oligosaccharide units. Likewise, in a study supporting this result, the role of mannose oligosaccharide in the adhesion and invasion of LF82 bacteria was investigated by cell infection experiments using the I-407 cell line. The results showed that mannose strongly inhibited LF82 invasion and that inhibition largely occurred via type-1 pili/mannosyl interaction (18). In another study, commercially purchased mucin was added to the cell culture medium at a certain rate before it was infected with $E$. coli $\mathrm{C} 25$ strain. The results obtained after the infection period showed that mucin inhibited bacterial translocation strongly in both cell lines and the bacterial pili retained by the mucin-bound oligosaccharide units without reaching the cell (19). On the other hand, it was quite remarkable that the MucMIR medium originating from the ileum inhibited more LF82 invasion than the Muc-MCR medium prepared from the colon region of intestine. Similarly, goblet cells in both the ileum and the colon secrete the same mucin called MUC-2. Thus, these results support the view that the mucin in the colon and the small intestine may exhibit different properties although they have the same structure (10-12). In addition, it is suggested that specific receptor-ligand interactions with LF82 strain may occur, because medias originated from colon and ileum regions of intestine affect LF82 invasion in different ratios. Also, oligosaccharide units associated with mucin may play a decisive role in the distribution of gut flora in the gut system (13). If these interactions can be identified in further studies, specific molecules can be identified that facilitate the detection of LF82.

\section{Conclusion}

In conclusion, this study showed that AIEC LF82 strain has different patterns in terms of the adhesion and invasion capabilities in mucosal medium originated from ileum region of healthy FBV/N female mice strain. Therefore, our data have a potential importance to give new ideas about determining the localization of AIEC bacteria within the healthy intestinal mucosa as in our previous work (14).

\section{Acknowledgement}

The authors thank all members of MISH (Universite' d'Auvergne, Clermont-Ferrand, France), especially Dr. Barnich and Dr. Billard for their great supports. We also thank the ERASMUS Traineeship Program 2015.

\section{Ethics}

Ethics Committee Approval: Animal protocols were performed according to the local ethical rules during ERASMUS+
Traineeship Program between $23^{\text {th }}$ and June $19^{\text {th }} 2015$ in Universite ' d'Auvergne (Clermont-Ferrand, France).

Informed Consent: In vitro study.

Peer-review: Externally and internally peer-reviewed.

\section{Authorship Contributions}

Concept: H.A., F.U., Design: H.A., M.K., Data Collection or Processing: H.A., M.K., Analysis or Interpretation: H.A., F.U., M.K., Literature Search: H.A., F.U., M.K., Writing: M.K.

Conflict of Interest: No conflict of interest was declared by the authors.

Financial Disclosure: The authors declared that this study received no financial support.

\section{References}

1. Rutgeerts P, Goboes K, Peeters M, Hiele M, Penninckx F, Aerts R, et al. Effect offaecal stream diversion on recurrence of Crohn's disease in the neoterminal ileum. Lancet 1991;338:771-4.

2. Sartor RB. Microbial influences in inflammatory bowel diseases. Gastroenterology 2008;134:577-94.

3. Schultsz C, Moussa M, van Ketel R, Tytgat GN, Dankert J. Frequency of pathogenic and enteroadherent Escherichiacoli in patients with inflammatory bowel disease and controls. J Clin Pathol 1997;50:573-9.

4. Baumgart M, Dogan B, Rishniw M, Weitzman G, Bosworth B, Yantiss R, et al. Culture independent analysis of ileal mucosa reveals a selective increase in invasive Escherichia coli of novel phylogeny relative to depletion of Clostridiales in Crohn's disease involving the ileum. ISME J 2007;1:403-18.

5. Kotlowski R, Bernstein CN, Sepehri S, Krause DO. High prevalence of Escherichia coli belonging to the $\mathrm{B} 2+\mathrm{D}$ phylogenetic group in inflammatory bowel disease. Gut 2007;56:669-75.

6. Boudeau J, Glasser AL, Masseret E, Joly B, Darfeuille-Michaud A. Invasive Ability of an Escherichia coli Strain Isolated from the Ileal Mucosa of a Patient with Crohn's Disease. Infect Immun 1999;67:4499-509.

7. Carvalho FA, Barnich N, Sauvanet P, Darcha C, Gelot A, DarfeuilleMichaud A. Crohn's disease-associated Escherichia coli LF82 aggravates colitis in injured mouse colon via signaling by flagellin. Inflamm Bowel Dis 2008;14:1051-60.

8. Johansson ME, Ambort D, Pelaseyed T, Schütte A, Gustafsson JK, Ermund A, et al. Composition and functional role of the mucus layers in the intestine. Cell Mol Life Sci 2011;68:3635-41.

9. Lang T, Hansson GC, Samuelsson T. Gel-forming mucins appeared early in metazoan evolution. Proc Natl Acad Sci U S A 2007;104:16209-14.

10. Audie JP, Janin A, Porchet N, Copin MC, Gosselin B, Aubert JP. Expression of human mucin genes in respiratory, digestive, and reproductive tracts as certained by in situ hybridization. J Histochem Cytochem 1993;41:1479-85.

11. Weiss AA, Babyatsky MW, Ogata S, Chen A, Itzkowitz SH. Expression of MUC2 and MUC3 mRNA in human normal, malignant, and inflammatory intestinal tissues. J Histochem Cytochem 1996;44:1161-6. 
12. Hansson GC, Johansson ME. The inner of the two Muc2 mucin dependent mucus layers in colon is devoid of bacteria. Gut Microbes 2010;1:51-4.

13. Larsson JM, Karlsson H, Sjovall H, Hansson GC. A complex, but uniform O-glycosylation of the human MUC2 mucin from colonic biopsies analyzed by nanoLC/MSn. Glycobiology 2009;19:756-66.

14. Aygun H, Karamese M, Ozic C, Uyar F. The effects of mucosal media on some pathogenic traits of Crohn's disaese-associated Escherichia coli LF82. Future Microbiol 2018;13:141-9.

15. Darfeuille-Michaud A, Boudeau J, Bulois P, Neut C, Glasser AL, Barnich N, et al. High prevalence of adherent-invasive Escherichia coli associated with ileal mucosa in Crohn's disease. Gastroenterology 2004;127:412-21.

16. Seksik P, Rigottier-Gois L, Gramet G, Sutren M, Pochart P, Marteau $\mathrm{P}$, et al. Alterations of the dominant faecal bacterial groups in patients with Crohn's disease of the colon. Gut 2003;52:237-42.
17. Vasquez N, Mangin I, Lepage P, Seksik P, Duong JP, Blum S, et al. Patchy distribution of mucosal lesions in ileal Crohn's disease is not linked to differences in the dominant mucosa-associated bacteria: a study using fluorescence in situ hybridization and temporal temperature gradient gel electrophoresis. Inflamm Bowel Dis 2007;13:684-92.

18. Boudeau J, Barnich N, Darfeulle-Michaud A. Type-1 pili-mediated adherence of Escherichia coli strain LF82 isolated from Crohn's disease is involved in bacterial invasion of intestinal epithelial cells. Mol Microbiol 2001;39:1272-84.

19. Gork AS, Usui N, Ceriati E, Drongowski RA, Epstein MD, Coran AG, et al. The effect of mucin on bacterial translocation in I-407 fetal and Caco-2 adult enterocyte cultured cell lines. Pediatr Surg Int 1999;15:155-9. 